\title{
Synchronization of Chimera States in Coupled Networks of Nonlinear Chaotic Oscillators
}

\author{
A. V.Bukh, G. I. Strelkova, V.S. Anishchenko
}

Effects of synchronization of chimera states are studied numerically in a two-layer network of nonlocally coupled nonlinear chaotic discrete-time systems. Each layer represents a ring of nonlocally coupled logistic maps in the chaotic mode. A control parameter mismatch is introduced to realize distinct spatiotemporal structures in isolated ensembles. We consider external synchronization of chimeras for unidirectional intercoupling and mutual synchronization in the case of bidirectional intercoupling. Synchronization is quantified by calculating the crosscorrelation coefficient between the symmetric elements of the interacting networks. The same quantity is used to determine finite regions of synchronization inside which the cross-correlation coefficient is equal to 1 . The identity of synchronous structures and the existence of finite synchronization regions are necessary and sufficient conditions for establishing the synchronization effect. It is also shown that our results are qualitatively similar to the synchronization of periodic self-sustained oscillations.

Keywords: multilayer networks, nonlocal coupling, chimera states, synchronization

Received September 19, 2018

Accepted October 22, 2018

This work was supported by DFG in the framework of SFB 910, by the Russian Ministry of Education and Science (Project Code 3.8616.2017) and by the Russian Science Foundation (grant No. 16-12-10175).

Andrei V.Bukh

buh. andrey@yandex.ru

Galina I. Strelkova

strelkovagi@info.sgu.ru

Vadim S. Anishchenko

wadim@info.sgu.ru

Saratov State University

ul. Astrakhanskaya 83, Saratov, 410012 Russia

RUSSIAN JOURNAL OF NONLINEAR DYNAMICS, 2018, 14(4), 419-433 


\section{Introduction}

In the past few decades, exploring the collective dynamics of complex systems of different nature, synchronization of ensembles of interacting oscillators and dissipative structure formation have been in the focus of great attention of many researchers in nonlinear dynamics. A lot of monographs [1-4] and papers [5-11] are devoted to this research topic. It has been established that, when interacting, nonlinear ensembles and networks can typically demonstrate various interesting effects, such as synchronization cluster formation, spatial intermittency, spatially stationary (motionless) regular and chaotic structures, etc. The majority of works deal, as a rule, with ensembles of identical oscillatory elements with local or global coupling between them. Recently, a novel type of spatiotemporal structures, called a chimera state [12], has been discovered in ensembles of nonlocally coupled identical phase oscillators. Nonlocal coupling means that each ensemble oscillator is connected with a finite number of neighbors to the left and right. Later this pattern was explored in detail in the papers $[13,14]$ where the term "chimera state" was proposed. Chimera denotes such a dynamical state when the network splits into coexisting domains with coherent (synchronous) and incoherent (asynchronous) dynamics of the network elements and these clusters are clearly spatially localized across the network.

We note that chimera-like structures were observed long before their definition in [13]. The attention of researchers in those years was aimed at the analysis of transitions from spatiotemporal chaos to complete synchronization of ensemble elements $[15,16]$. Chimera-like patterns were found to be transient to the synchronization regime and were called synchronous and desynchronous clusters (see, for example, [17, 18]). Recently, the discovery of chimera states attracted much attention and aroused a great interest of many researchers and led to the growth of numerical and theoretical [19-43, 45, 46] and then experimental [47-52] studies. The analysis of various spatiotemporal patterns (including chimeras) in complex ensembles is not only of fundamental scientific, but also of great practical significance. This is particularly important in exploring arrays of Josephson junctions [53], large arrays of coupled lasers [54], neural networks [55], brain dynamics [56], power grids [57, 58], etc.

Real-world networks, however, are typically not isolated and always functionally and conditionally connected to other networks. It is therefore important to explore the dynamics of coupled or multilayer networks [59-63]. In this case synchronization of various spatiotemporal patterns in such systems becomes one of the main and intriguing research topics. This problem was studied before for coupled dynamical systems [6, 64-67], but basically for coupled identical oscillators with local or global coupling. Moreover, synchronous structures and synchronization regions were not quantitatively estimated and defined in the parameter space. Recently, a number of works have appeared which are devoted to synchronization between coupled complex networks [68-73]. However, there are only few papers where it has been shown that chimera states can be synchronized across networks [72, 73].

The objective of our paper is to study effects of external and mutual synchronization of various complex spatiotemporal structures, including chimera states, in a two-layer network made of coupled ensembles of nonlocally coupled chaotic discrete-time systems. The individual elements in each subnetwork are described by the well-known logistic map in the chaotic regime. The logistic map is the simplest and famous example of a wide class of nonlinear chaotic systems with the Feigenbaum scenario of chaos onset. Moreover, it has been shown that an ensemble of nonlocally coupled logistic maps in the chaotic regime can realize chimera states $[25,26]$. In our study we introduce a control parameter mismatch in each subnetwork, which enables us to implement various spatiotemporal structures, including chimeras, in the interacting ensembles 
when there is no coupling between them. In order to establish and prove the synchronization effects, the identity of synchronous patterns is quantified by calculating the cross-correlation coefficient between relevant (symmetric) elements of the coupled ensembles. This quantity is also used to estimate and plot the synchronization regions in the planes of different parameters of the two-layer network of interest.

\section{Model under study}

We consider the dynamics of the two-layer multiplex network consisting of nonlocally coupled nonlinear chaotic oscillators, which is schematically shown in Fig. 1.

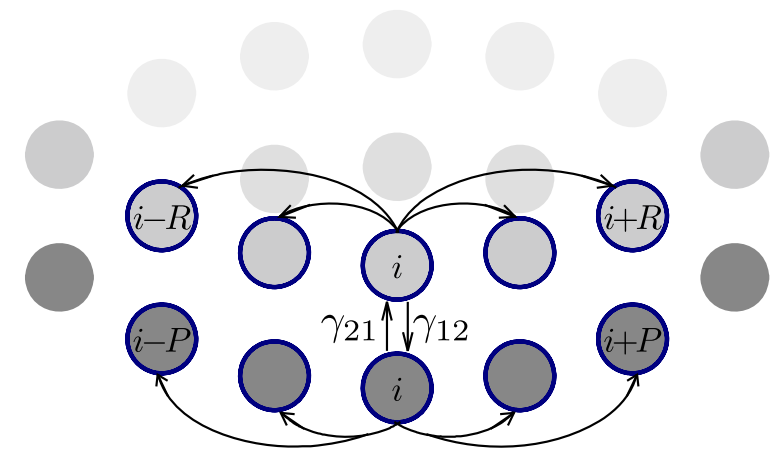

Fig. 1. Schematic representation of a two-layer multiplex network. Each layer is a ring of nonlocally coupled oscillators. The $i$ th oscillators $(i=1,2, \ldots, N)$ of the interacting rings are coupled with coupling coefficients $\gamma_{21}$ and $\gamma_{12}$.

The model under study is described by the following system of equations:

$$
\begin{aligned}
& x_{i}^{t+1}=f_{i}^{t}+\frac{\sigma_{1}}{2 R} \sum_{j=i-R}^{i+R}\left[f_{j}^{t}-f_{i}^{t}\right]+\gamma_{21} F_{i}^{t}, \\
& y_{i}^{t+1}=g_{i}^{t}+\frac{\sigma_{2}}{2 P} \sum_{j=i-P}^{i+P}\left[g_{j}^{t}-g_{i}^{t}\right]+\gamma_{12} G_{i}^{t},
\end{aligned}
$$

where $x_{i}^{t}$ and $y_{i}^{t}$ are real dynamical variables of coupled ensembles, $i$ is the number of the ensemble element, $i=1,2, \ldots, N, N$ is the total number of elements in each subnetwork and is equal to $1000, t$ denotes the discrete time. In our study the individual elements in both ensembles are defined by the logistic map $f_{i}^{t}=\alpha_{1} x_{i}^{t}\left(1-x_{i}^{t}\right)$ and $g_{i}^{t}=\alpha_{2} y_{i}^{t}\left(1-y_{i}^{t}\right)$ with different control parameters $\alpha_{1}$ and $\alpha_{2} . \sigma_{1}$ and $\sigma_{2}$ specify the nonlocal coupling strengths (intracouplings) in the rings. $R$ and $P$ denote the number of neighbors of the $i$ th element to each side in the first and second subsystems, respectively. These parameters are also known to be the measure of nonlocal interaction of the elements in isolated ensembles and define the coupling range. The subnetworks in (2.1) are connected through the coupling functions $F_{i}^{t}=\left(g_{i}^{t}-f_{i}^{t}\right)$ and $G_{i}^{t}=\left(f_{i}^{t}-g_{i}^{t}\right)$ which denote the intercoupling. $\gamma_{12}$ and $\gamma_{21}$ are the intercoupling strengths.

In order to analyze synchronization effects, we introduce a control parameter detuning in both rings by setting $\alpha_{1}=3.7$ and $\alpha_{2}=3.85$ which correspond to the chaotic regime in the individual elements of the first and second subnetworks, respectively. We also choose different intracouplings between the elements in each ring as $\sigma_{1}=0.23$ and $\sigma_{2}=0.15$. We fix the equal 
number of neighbors $P=R=320$, at which the uncoupled subnetworks can show chimera states $[25,26,43]$.

Each subnetwork in the system (2.1) is highly sensitive to the change in the parameters $\alpha_{1,2}$ and $\sigma_{1,2}$. When the subnetworks are uncoupled $\left(\gamma_{12}=\gamma_{21}=0\right)$, a slight variation of $\alpha_{1,2}$ and $\sigma_{1,2}$ causes a significant difference in the spatiotemporal patterns realized in the rings. The parameter mismatch provides completely different spatiotemporal structures in the $x_{i}^{t}$ and $y_{i}^{t}$ ensembles when there is no coupling between them. This fact enables one to explore the effects of synchronization when the subnetworks under study are coupled.

Equations (2.1) are solved numerically for periodic boundary conditions (we consider the ring network scheme) and for initial conditions $\left(x_{i}^{0}, y_{i}^{0}\right)$ randomly distributed across the network nodes $i=1,2, \ldots, N$ within the interval $[0 ; 1]$. The total iteration time is $6 \times 10^{5}$ and a transient of the first $5 \times 10^{5}$ iterations is discarded.

\section{External synchronization}

We start with considering external synchronization in the model (2.1). In this case the intercoupling is unidirectional, i.e., $\gamma_{21}=0$ and $\gamma_{12}=\gamma>0$. This means that the nodes in the first (driver) network are coupled unidirectionally to the nodes in the second (response) network. A similar scheme of the drive-response network pairs was considered in $[68,69,72]$.

In our studies the values of $\alpha_{1,2}, \sigma_{1,2}, P$ and $R$ are chosen in such a way that at least one of the subnetworks shows a chimera state when there is no coupling between the subnetworks. The spatiotemporal patterns which are realized in the isolated rings for the chosen parameter values are exemplified in Fig. 2 by space-time profiles for the amplitudes $x_{i}^{t}$ and $y_{i}^{t}$ of each ring. A space-time profile was proposed in [43] and represents a set (collection) of a finite number of snapshots of the network dynamics and thus visualizes the temporal dynamics of the whole network. All the space-time profiles shown in the present paper consist of 100 snapshots taken during the first 100 iterations after the transient time. We point out that an alternative graphical representation in the form of recurrence plots has been proposed in [44] to identify chimera states.

As can be seen from Fig. 2, the driver network $x_{i}^{t}$ shows amplitude and phase chimeras $[25,43]$ (Fig. 2a), and the second ring $y_{i}^{t}$ demonstrates spatiotemporal chaos (Fig. 2b). We note that all the chimeras and the other spatiotemporal patterns obtained in our work are permanent and not transient. When the unidirectional coupling with strength $\gamma>0$ is introduced, the influence

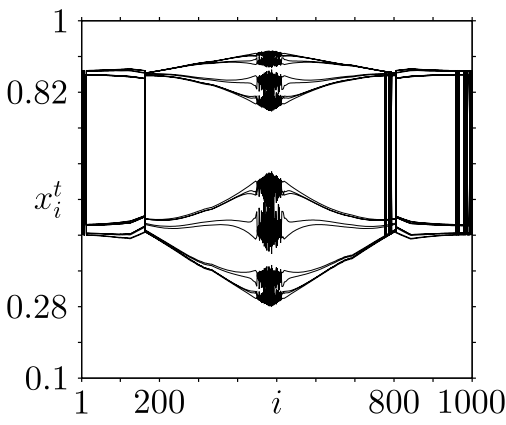

(a)

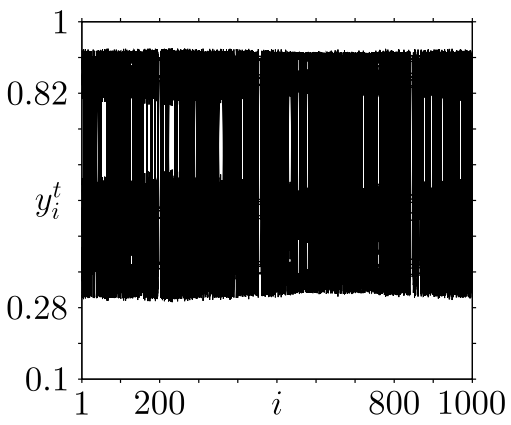

(b)

Fig. 2. Space-time profiles of amplitudes $x_{i}^{t}$ (a) and $y_{i}^{t}$ (b) in the network (2.1) without coupling $(\gamma=0)$ and for $\alpha_{1}=3.7, \alpha_{2}=3.85, \sigma_{1}=0.23$, and $\sigma_{2}=0.15$. 
of the driver network induces the appearance of amplitude and phase chimeras in the response network. However, if the intercoupling is sufficiently weak, e.g., $\gamma=0.15$ and $\gamma=0.3$, the space-time profile of $y_{i}^{t}$ does not coincide yet completely with that of $x_{i}^{t}$ at $\gamma=0$ (Fig. 2a). The corresponding results for the dynamics of the response network $y_{i}^{t}$ are shown in Fig. 3.

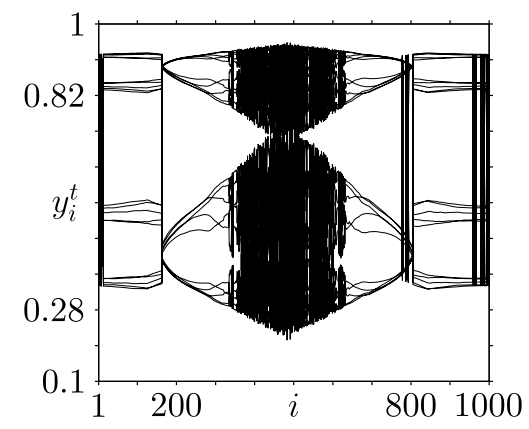

(a)

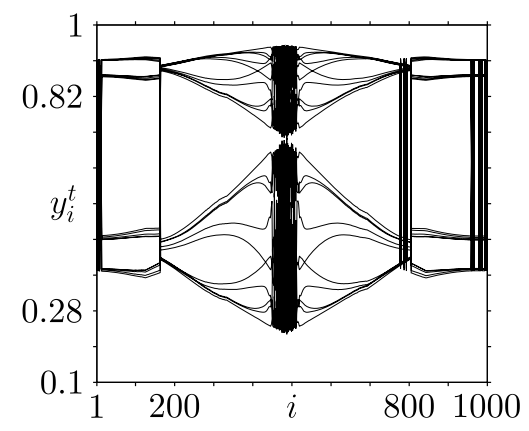

(b)

Fig. 3. Space-time profiles of dynamical states $y_{i}^{t}$ of the response network in (2.1) when affected by the driver with the intercoupling strength $\gamma=0.15$ (a) and $\gamma=0.3$ (b). Other parameters are as in Fig. 2.

If the intercoupling strength increases further, $\gamma \geqslant 0.4$, the structures in both rings become rather identical (Fig. 4) and there are only minor differences in their amplitudes (coordinates' values).

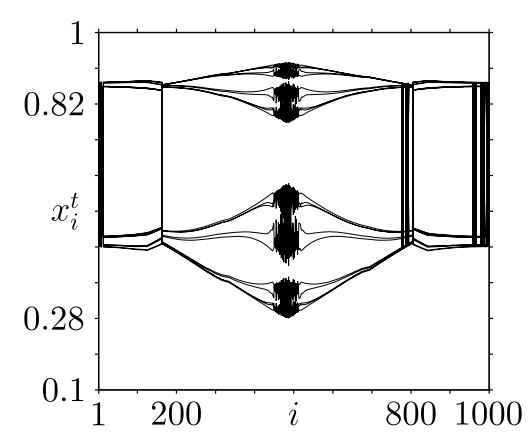

(a)

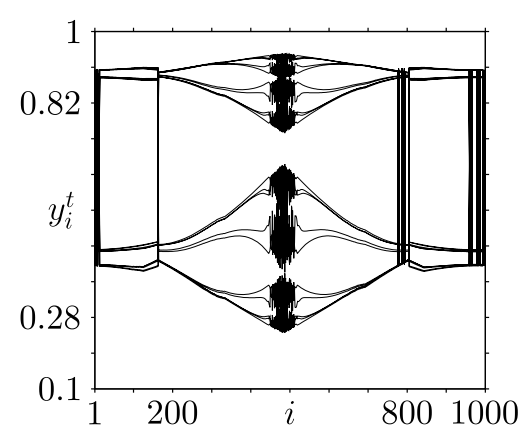

(b)

Fig. 4. Space-time profiles of the $x_{i}^{t}$ (a) and $y_{i}^{t}$ (b) coordinates of the network (2.1) in the synchronization mode at $\gamma=0.45$. Other parameters are as in Fig. 2.

In order to conclude that the external synchronization takes place indeed, two important facts must be justified. We need to quantify the identity of synchronous structures (Figs. 4a and $4 \mathrm{~b}$ ) and to show that there is a finite region of synchronization in the parameter plane of the system (2.1). To justify the identity of the synchronous patterns presented in Figs. 4a and $4 \mathrm{~b}$ we need to show that elements with the number $i(i=1,2, \ldots, N)$ of the $x_{i}^{t}$ and $y_{i}^{t}$ ensembles oscillate synchronously in time. This can be done by calculating the cross-correlation coefficient $R_{i}$ between relevant elements of the coupled networks as follows:

$$
\begin{gathered}
R_{i}=\frac{\left\langle\widetilde{x}_{i}(t) \widetilde{y}_{i}(t)\right\rangle}{\sqrt{\left\langle\widetilde{x}_{i}^{2}(t)\right\rangle\left\langle\widetilde{y}_{i}^{2}(t)\right\rangle}} \\
\widetilde{x}_{i}(t)=x_{i}(t)-\left\langle x_{i}(t)\right\rangle, \\
\widetilde{y}_{i}(t)=y_{i}(t)-\left\langle y_{i}(t)\right\rangle .
\end{gathered}
$$




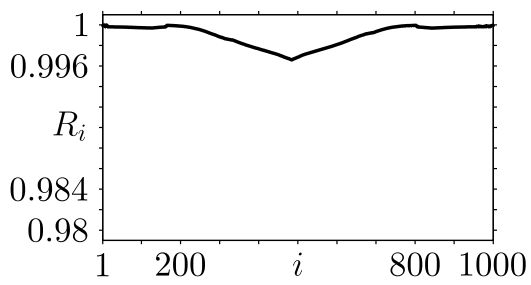

Fig. 5. Cross-correlation coefficient $R_{i}$ (3.1) calculated for the structures in Fig. 4 at $\gamma=0.45$. Other parameters are as in Fig. 2.

The angle brackets $\langle\cdot\rangle$ in (3.1) mean the time averaging. If spatiotemporal structures are identical, the cross-correlation coefficient $R_{i}$ must be equal to 1 . The numerical results for $R_{i}$ are plotted in Fig. 5 for the regimes presented in Fig. $4 \mathrm{a}$ and $4 \mathrm{~b}$. It is clearly seen that $0.99<R_{i} \leqslant 1.0$ for all $i=1,2, \ldots, N$. This implies that the observed structures are identical or synchronous. We note that synchronous structures can also be quantitatively compared by using the recurrence quantification analysis [44].

The synchronization effect (Figs. 4 and 5) can also be well illustrated by computing time series $x_{i}^{t}(t)$ and $y_{i}^{t}(t)$ for relevant (symmetric) elements of the coupled subnetworks. The corresponding numerical results are presented in Fig. 6 for the $i=300$ th oscillators of the model (2.1). As follows from the plots, the oscillations are rather synchronous and are characterized by only a minor difference in the amplitude values, which is quite acceptable for synchronous regimes.

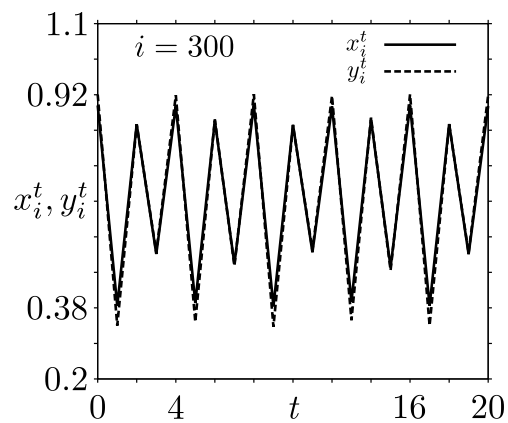

Fig. 6. Time series $x_{i}^{t}(t)$ and $y_{i}^{t}(t)$ for the 300th oscillators, calculated in the synchronization mode in the network (2.1) at $\gamma=0.45$. Other parameters are as in Fig. 2.

Thus, calculating the cross-correlation coefficient (Fig. 5), the space-time profiles (Fig. 4) and the time series $x_{i}^{t}(t)$ and $y_{i}^{t}(t)$ (Fig. 6) for the synchronous structures in the coupled subnetworks (2.1) allows us to assume that in the synchronization mode the condition $x_{i}^{t} \simeq y_{i}^{t}$ is satisfied for all $i=1,2, \ldots, N$ and over the observation time.

\section{Regions of external synchronization}

We now intend to show that the effect of external synchronization can be observed within finite ranges of the system parameter variation. In order to construct synchronization regions of spatiotemporal structures in the network (2.1), we consider two different cases. Firstly, we leave the control parameter in the driver network $\alpha_{1}=3.7$ fixed and vary the control parameter $\alpha_{2}$ in the response network. When the networks considered are uncoupled, varying $\alpha_{2}$ can result in 
the appearance of various spatiotemporal patterns in the second ring, including chimera states and periodic structures.

Let us analyze what happens with the above-mentioned patterns when the driver network $x_{i}^{t}$ influences unidirectionally the response network $y_{i}^{t}$ with $\gamma>0$ and when $\alpha_{2}$ is varied. Our calculations show that starting from $\gamma=0.4$, the driver network can induce a structure in the response network, which completely coincides with that in the driver ensemble (Fig. 4). The resulting synchronous regime is stable and remains unchanged within a finite region of synchronization as the parameter $\alpha_{2}$ is varied. The corresponding numerical results for the synchronization region is shown in Fig. 7 for the case when the unidirectional intercoupling $\gamma=0.4$. As a quantitative criterion for the existence of a finite synchronization region, we again use the calculated values for the cross-correlation coefficient (3.1). The borderlines of synchronization regions are defined by the condition $R_{i}>0.99$. The lack of synchronization and thus the escape from the synchronization regions correspond to the inequality $R_{i}<0.99$.

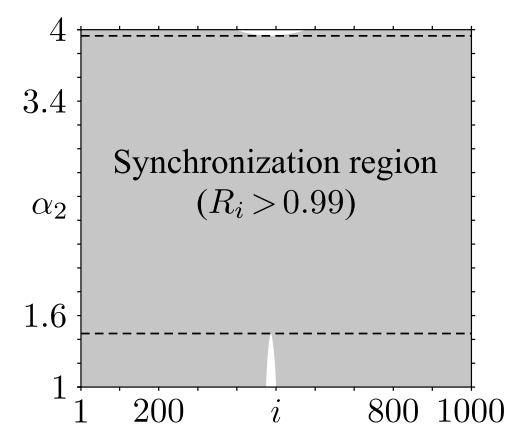

Fig. 7. External synchronization region (bounded by the dotted lines) for varying parameter $\alpha_{2}$ and for $\gamma=0.4, \alpha_{1}=3.7, \sigma_{1}=0.23$, and $\sigma_{2}=0.15$.

As follows from Fig. 7, at $\gamma=0.4$ there is a finite range (gray color) of values of parameter $\alpha_{2}$ $\left(1.45 \leqslant \alpha_{2} \leqslant 3.9\right)$, which is bounded by the horizontal dotted lines, and within this region the cross-correlation $R_{i}>0.99$ for all the elements of the network (2.1). Thus, we can conclude that the external synchronization is established. Inside this domain the response network shows the spatiotemporal pattern which is completely identical or synchronous to the structure in the driving network. The state $y_{i}^{t}$ of the response subsystem is fully determined by the driver dynamics $x_{i}^{t}$, i.e., the external synchronization is clearly observed. The domains above and below the bounded region in Fig. 7 correspond to the loss of synchronization $\left(R_{i}<0.99\right)$ by a part of the network elements.

We now turn to the second case. We fix the control parameter in the driven network as $\alpha_{2}=3.85$ and vary $\alpha_{1}$ in the driver at $\gamma=0.4$. When $\alpha_{1}$ changes, the first ring $x_{i}^{t}$ exhibits various spatiotemporal structures which are essentially different from the pattern observed in the second ring $y_{i}^{t}$ without intercoupling. When the two rings are coupled, the response network shows spatiotemporal structures which are synchronous with those realized in the driver network at given values of $\alpha_{1}$. In other words, the unidirectional intercoupling causes the driven network to fully follow and repeat the behavior of the driver network. Let us clarify this effect in more detail. Figure 8 depicts the synchronization region for the fixed $\alpha_{2}=3.85$ and $\gamma=0.4$ and when $\alpha_{1}$ is varied. The synchronization region is bounded by the horizontal dotted lines at $\alpha_{1}=3.5$ and $\alpha_{1}=3.9$. Inside this region the cross-correlation coefficient $R_{i}$ is larger than 0.99 and this clearly indicates the existence of external synchronization. Outside the synchronization region the spatiotemporal structures are desynchronized and $R_{i}<0.99$.

RUSSIAN JOURNAL OF NONLINEAR DYNAMICS, 2018, 14(4), 419-433 


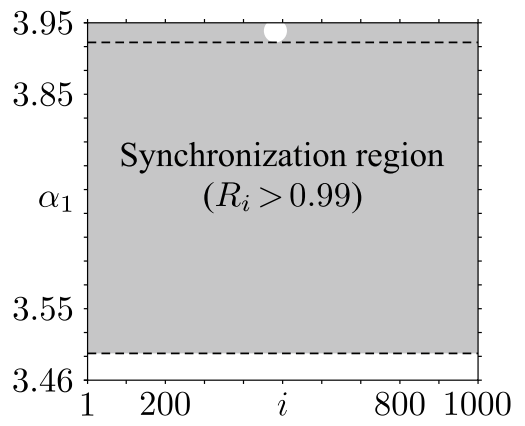

Fig. 8. Region of external synchronization (bounded by the horizontal lines at $\alpha_{1}=3.5$ and $\alpha_{1}=3.9$ ) in the network (2.1) depending on the parameter $\alpha_{1}$ for $\gamma=0.4, \alpha_{2}=3.85, \sigma_{1}=0.23$, and $\sigma_{2}=0.15$.
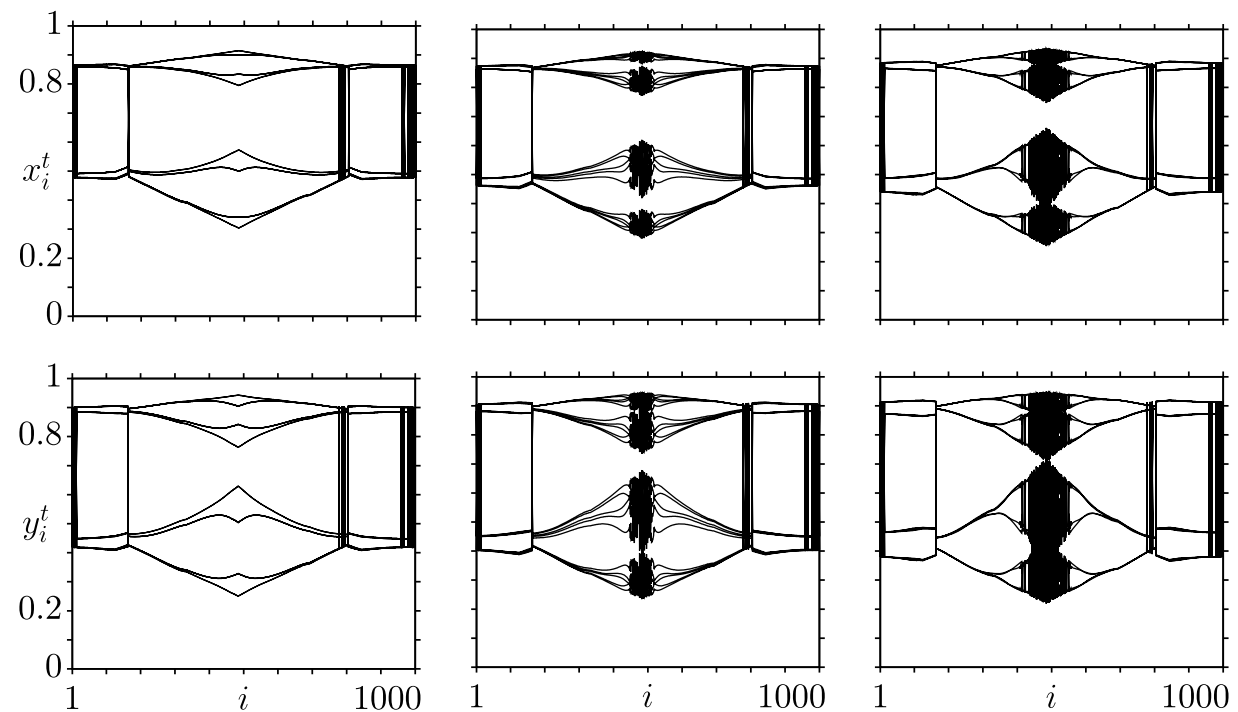

(a)

(b)

(c)

Fig. 9. Space-time profiles of synchronous spatiotemporal structures in the coupled rings $x_{i}^{t}$ (upper panel) and $y_{i}^{t}$ (low panel) for different values of $\alpha_{1}: 3.66$ (a), 3.7 (b), 3.75 (c) and for $\gamma=0.4, \alpha_{2}=3.85$, $\sigma_{1}=0.23$, and $\sigma_{2}=0.15$.

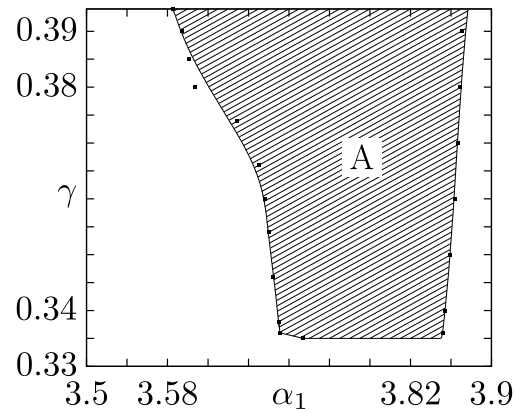

Fig. 10. Region of external synchronization of spatiotemporal structures in the network (2.1) in the $\left(\alpha_{1}, \gamma\right)$ parameter plane for $\alpha_{2}=3.85, \sigma_{1}=0.23$, and $\sigma_{2}=0.15$. 
As already mentioned, different values of $\alpha_{1}$ in the driving network are associated with various spatiotemporal patterns which induce identical synchronous structures in the response network. Figure 9 exemplifies typical structures which are realized in both rings $x_{i}^{t}$ and $y_{i}^{t}$ for different values of $\alpha_{1}$. Indeed, when $\alpha_{1}$ is varied, the spatiotemporal patterns change their form but remain identical and synchronous inside the synchronization region. In this case the spatiotemporal patterns in the response network $y_{i}^{t}$ "are locked" by the structures in the driver network $x_{i}^{t}$. The resulting patterns are synchronous as $R_{i}>0.99$.

Our numerical studies have shown that, as $\alpha_{1}$ is varied, the width of synchronization region depends on the intercoupling strength $\gamma$. To illustrate this dependence, the region of external synchronization of spatiotemporal structures in the model (2.1) is depicted in Fig. 10 in the $\left(\alpha_{1}, \gamma\right)$ parameter plane.

As can be clearly seen from the figure, the synchronization region has a tongue-like form, is characterized by a certain threshold value $\gamma_{\text {th }} \approx 0.333$ and expands in the parameter $\alpha_{1}$ as the intercoupling $\gamma$ increases. Our calculations have shown that the presence of the synchronization threshold $\gamma_{\text {th }}$ is due to the nonlocal coupling topology and its height depends on the coupling range (on the number of neighbors $R$ and $P$ in the model (2.1)).

\section{Mutual synchronization}

We return to the network (2.1) with the initially chosen parameter values: $\alpha_{1}=3.7$, $\alpha_{2}=3.85, P=R=320, N=1000$ and set the equal value of nonlocal coupling strengths in both rings as $\sigma_{1}=\sigma_{2}=0.28$. For the chosen values of $\alpha_{1,2}, \sigma_{1,2}$ and without the intercoupling, the $x_{i}^{t}$ ring demonstrates a spatio-periodic pattern, while the $y_{i}^{t}$ ring exhibits a chimera state.

We now intend to explore mutual synchronization between the two coupled rings. For this purpose we introduce the symmetric (bidirectional) intercoupling between the ensembles $\gamma_{12}=\gamma_{21}=\gamma$ and analyze numerically the evolution of spatiotemporal structures in the network (2.1) when the intercoupling strength is varied in the interval $[0,0.8]$. We iterate the network (2.1) during $1.2 \times 10^{6}$ in total and discard the first $10^{6}$ iterations as a transient.

The calculation results are presented in Fig. 11 where space-time profiles for the coordinates of each subnetwork are shown for three different values of the intercoupling strength. The spacetime profile represents a set of 100 snapshots taken within the time interval $10^{6} \div 10^{6}+100$. As can be seen from Fig. 11c, the spatiotemporal patterns are synchronized at $\gamma=0.075$ and this is corroborated by calculating the cross-correlation coefficient $R_{i}$ which is larger than 0.99 in this case. Thus, the mutual synchronization is established in the network (2.1). It is important to point out that the structures $x_{i}^{t}$ and $y_{i}^{t}$ synchronized at $\gamma=0.075$ differ from the corresponding patterns which are realized in these ensembles when there is no coupling between them. When the intercoupling is introduced between the rings, they are "tuned" to each other and mutually adjust the resulting synchronous spatiotemporal pattern. Such a behavior of interacting systems is rather typical in the case of mutual synchronization.

The effect of mutual synchronization takes place within a finite region of synchronization, which is depicted in Fig. 12 and is bounded by the horizontal dotted lines. As follows from this figure, the cross-correlation coefficient $R_{i} \geqslant 0.99$ inside the finite range of the intercoupling strength, $0.075 \leqslant \gamma \leqslant 0.72$, and thus indicates the existence of a finite region of mutual synchronization of spatiotemporal structures in the network (2.1).

RUSSIAN JOURNAL OF NONLINEAR DYNAMICS, 2018, 14(4), 419-433 

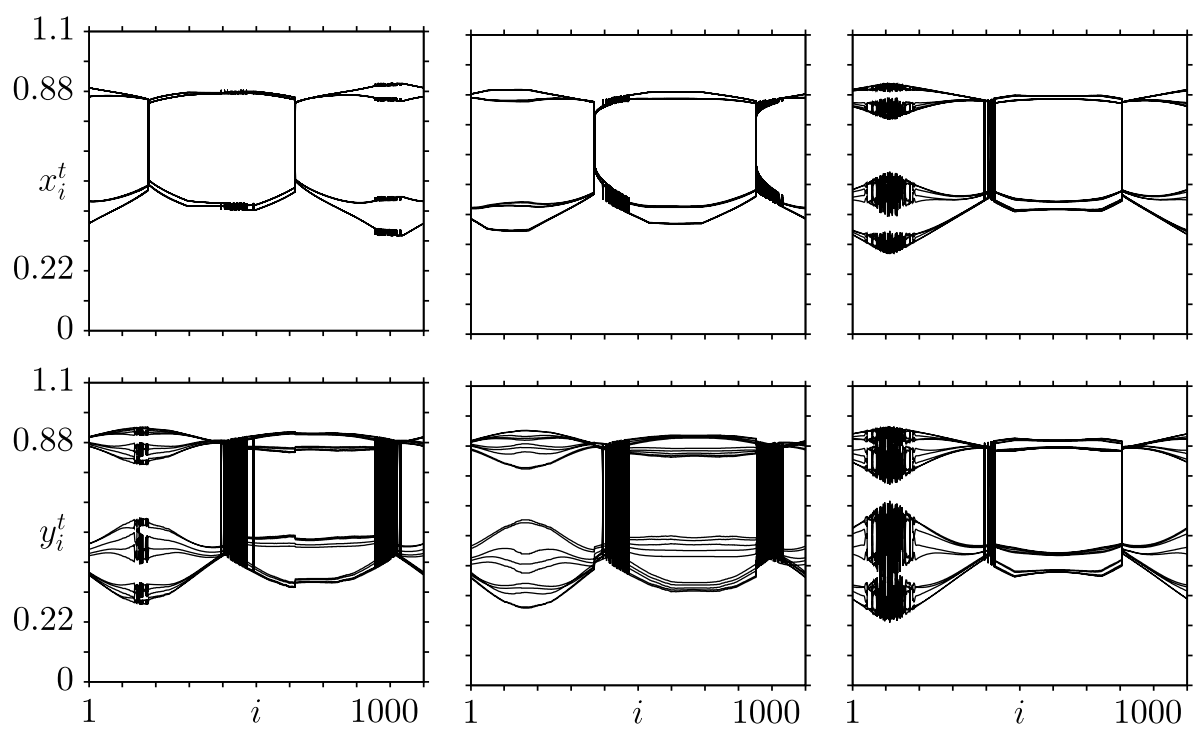

(a)

(b)

(c)

Fig. 11. Space-time profiles for the dynamics of symmetrically coupled ensembles $x_{i}^{t}$ (upper panel) and $y_{i}^{t}$ (low panel) for different values of intercoupling strength $\gamma: 0.011$ (a), 0.025 (b), and 0.075 (c) at $\alpha_{1}=3.7$, $\alpha_{2}=3.85, \sigma_{1}=\sigma_{2}=0.28$.

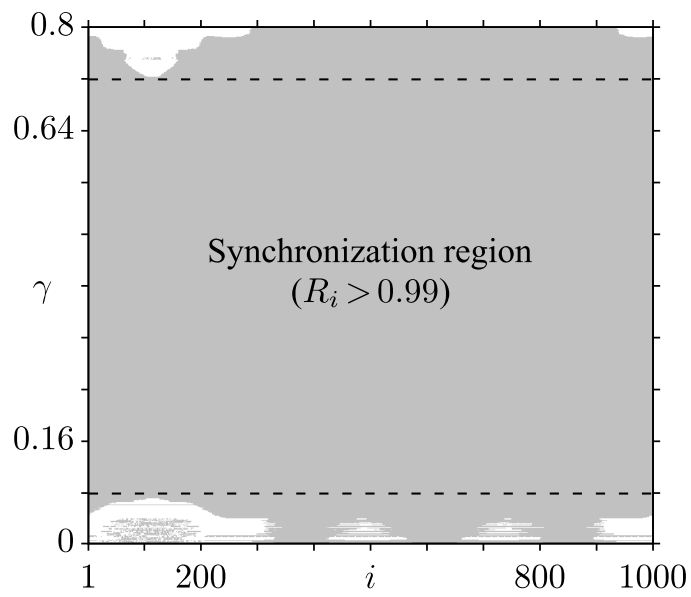

Fig. 12. Region of mutual synchronization (bounded by the horizontal dotted lines) of spatiotemporal structures in the network (2.1) when varying the intercoupling $\gamma$ and for $\alpha_{1}=3.7, \alpha_{2}=3.85$, and $\sigma_{1}=\sigma_{2}=0.28$.

\section{Discussion and conclusions}

In our work we have studied numerically the dynamics and synchronization phenomena in the two-layer network of nonlocally coupled chaotic discrete-time systems (2.1) with unidirectional and symmetrical couplings between the one-layer networks. Our results have confidently indicated that the effects of external and mutual synchronization of spatiotemporal structures, including chimera states, take place in the network considered. We have demonstrated that synchronized patterns are identical and observed within finite regions of synchronization, which are the necessary and sufficient conditions for realizing and justifying the synchronization effect. The identity of synchronous structures and the synchronization regions are estimated quantita- 
tively by calculating the cross-correlation coefficient $R_{i}(3.1)$ of oscillations of the $i$ th elements of the interacting ensembles, where $i=1,2, \ldots, N$.

The phenomenon of synchronization of spatiotemporal structures can be qualitatively compared with the classical effect of synchronization of periodic self-sustained oscillations $[4,18]$. In this case the spectral oscillation line at a frequency $\omega$ can be treated as the simplest structure. In the case of external synchronization the behavior of a driver oscillator is characterized by the spectral line at the frequency $\omega_{1}$; the structure of a driven oscillator, by the spectral line at the frequency $\omega_{0}$. When the external synchronization is established, a frequency locking occurs inside the synchronization region. This means that the driven oscillator frequency $\omega_{0}$ shifts and coincides with the driver oscillator frequency $\omega_{0}=\omega_{1}$, e.g., $\omega_{0}$ is locked by $\omega_{1}$. This frequency equality remains unchanged (constant) within the synchronization region. Varying $\omega_{1}$ causes $\omega_{0}$ to change so that the equality $\omega_{0}=\omega_{1}$ always holds inside the synchronization region. A similar effect takes place for mutual synchronization between two oscillators with different natural frequencies $\omega_{1}$ and $\omega_{2}$. The only difference is that, inside the synchronization region, the oscillations are generated either with frequency $\omega_{1}$ or $\omega_{2}$, or with a certain intermediate frequency $\omega_{1}<\omega<\omega_{2}$.

Our numerical results presented in the paper show that a qualitatively equivalent behavior occurs for the two-layer network of nonlocally coupled nonlinear discrete-time systems. In the case of external synchronization the spatiotemporal structure of the response network "is locked" by the structure of the driver network and the identical patterns remain unchanged within the synchronization region (see Figs. 4-7). When the mutual synchronization takes place, the spatiotemporal structures in the coupled one-layer networks mutually adjust the synchronous pattern. Moreover, the resulting synchronous structures differ from the initially observed patterns which are realized in the coupled rings without intercoupling (see Fig. 11). This feature is also very typical of mutual synchronization of coupled periodic self-sustained oscillators.

Finally, the region of external synchronization depicted in Fig. 10 is the most illustrative and bright result of our studies. This plot can be qualitatively compared with relevant pictures obtained in the framework of the classical theory of external synchronization of periodic self-sustained oscillations $[4,18]$. As is well known, the region of external synchronization is constructed on the "external signal amplitude - frequency mismatch (detuning)" parameter plane. The synchronization region narrows with decreasing external amplitude and vanishes at its zero value. In our case the intercoupling strength $\gamma$ plays the role of the external signal amplitude and defines the oscillation amplitude (the coordinate value) $y_{i}$ in the response network. At a fixed $\alpha_{2}=$ const, the parameter $\alpha_{1}$ determines a detuning (mismatch) of structures, $\Delta \alpha=\left|\alpha_{1}-\alpha_{2}\right|$. However, unlike the external synchronization of periodic self-sustained oscillations, there is a synchronization threshold in the intercoupling strength $\gamma$ when the two one-layer networks are unidirectionally coupled (see Fig. 10). We believe that this threshold effect is associated with a complicated topology of the inner couplings between the elements of the network (2.1).

The above comparison enables one to conclude that the results of this paper can be considered as a generalization of the notions of the classical phenomenon of synchronization of periodic self-sustained oscillations to the case of synchronization of spatiotemporal structures in multilayer networks of nonlocally coupled nonlinear oscillators. The effects of external and mutual synchronization which have been established and described in our work are general and can be observed for different values of the control parameters of individual elements of subnetworks as well as for different values of the coupling parameters. We also believe that our findings will considerably contribute to the synchronization of chimera states and various complex spatiotemporal structures in multilayer networks.

RUSSIAN JOURNAL OF NONLINEAR DYNAMICS, 2018, 14(4), 419-433 


\section{References}

[1] Afraimovich, V.S., Nekorkin, V.I., Osipov, G.V., and Shalfeev, V.D., Stability, Structures and Chaos in Nonlinear Synchronization Network, World Sci. Ser. Nonlinear Sci. Ser. A Monogr. Treatises, vol. 6, Singapore: World Sci., 1994.

[2] Nekorkin, V.I. and Velarde, M. G., Synergetic Phenomena in Active Lattices: Patterns, Waves, Solitons, Chaos, Berlin: Springer, 2002.

[3] Osipov, G., Kurths, J., and Zhou, C., Synchronization in Oscillatory Networks, Berlin: Springer, 2007.

[4] Pikovsky, A., Rosenblum, M., and Kurths, J., Synchronization: A Universal Concept in Nonlinear Sciences, New York: Cambridge Univ. Press, 2001.

[5] Nekorkin, V.I. and Makarov, V.A., Spatial Chaos in a Chain of Coupled Bistable Oscillators, Phys. Rev. Lett., 1995, vol. 74, no. 24, pp. 4819-4822.

[6] Nekorkin, V.I., Kazantsev, V.B., and Velarde, M. G., Mutual Synchronization of Two Lattices of Bistable Elements, Phys. Lett. A, 1997, vol. 236, nos. 5-6, pp. 505-512.

[7] Nekorkin, V.I., Voronin, M.L., and Velarde, M. G., Clusters in an Ensemble of Globally Coupled Bistable Oscillators, Eur. Phys. J. B, 1999, vol. 9, no. 3, pp. 533-543.

[8] Belykh, V. N., Belykh, I. V., and Hasler, M., Hierarchy and Stability of Partially Synchronous Oscillations of Diffusively Coupled Dynamical Systems, Phys. Rev. E (3), 2000, vol.62, no. 5, part A, pp. 6332-6345.

[9] Belykh, V.N., Belykh, I.V., and Mosekilde, E., Cluster Synchronization Modes in an Ensemble of Coupled Chaotic Oscillators, Phys. Rev. E, 2001, vol. 63, no. 3, 036216, 4 pp.

[10] Akopov, A., Astakhov, V., Vadivasova, T., Shabunin, A., and Kapitaniak, T., Frequency Synchronization of Clusters in Coupled Extended Systems, Phys. Lett. A, 2005, vol. 334, nos. 2-3, pp. 169-172.

[11] Pecora, L. M., Sorrentino, F., Hagerstrom, A. M., Murphy, Th. E., and Roy, R., Symmetries, Cluster Synchronization, and Isolated Desynchronization in Complex Networks, Nat. Commun., 2014, vol. 5, 4079, 8 pp.

[12] Kuramoto, Y. and Battogtokh, D., Coexistence of Coherence and Incoherence in Nonlocally Coupled Phase Oscillators, Nonlinear Phenomena in Complex Systems, 2002, vol. 5, no. 4, pp. 380-385.

[13] Abrams, D. M. and Strogatz, S. H., Chimera States for Coupled Oscillators, Phys. Rev. Lett., 2004, vol. 93, no. 17, 174102, 4 pp.

[14] Panaggio, M. J. and Abrams, D. M., Chimera States: Coexistence of Coherence and Incoherence in Networks of Coupled Oscillators, Nonlinearity, 2015, vol. 28, no. 3, R67-R87.

[15] Waller, I. and Kapral, R., Spatial and Temporal Structure in Systems of Coupled Nonlinear Oscillators, Phys. Rev. A, 1984, vol. 30, no. 4, pp. 2047-2055.

[16] Kaneko, K., Pattern Dynamics in Spatiotemporal Chaos: Pattern Selection, Diffusion of Defect and Pattern Competition Intermittency, Phys. D, 1989, vol. 34, nos. 1-2, pp. 1-41.

[17] Astakhov, V.V., Anishchenko, V.S., and Shabunin, A.V., Controlling Spatiotemporal Chaos in a Chain of the Coupled Logistic Maps, IEEE Trans. Circuits Syst. I, 1995, vol.42, no.6, pp. 352-357.

[18] Anishchenko, V.S., Astakhov, V.V., Neiman, A. B., Vadivasova, T.E., and Schimansky-Geier, L., Nonlinear Dynamics of Chaotic and Stochastic Systems: Tutorial and Modern Developments, 2nd ed., Berlin: Springer, 2007.

[19] Abrams, D. M., Mirollo, R., Strogatz, S.H., and Wiley, D. A., Solvable Model for Chimera States of Coupled Oscillators, Phys. Rev. Lett., 2008, vol. 101, no. 8, 084103, 4 pp.

[20] Laing, C. R., Chimeras in Networks of Planar Oscillators, Phys. Rev. E (3), 2010, vol. 81, no. 6, 066221, 4 pp.

[21] Laing, C. R., Fronts and Bumps in Spatially Extended Kuramoto Networks, Phys. D, 2011, vol. 240, no. 24, pp. 1960-1971. 
[22] Martens, E.A., Laing, C.R., and Strogatz, S.H., Solvable Model of Spiral Wave Chimeras, Phys. Rev. Lett., 2010, vol. 104, no. 4, 044101, 4 pp.

[23] Motter, A.E., Nonlinear Dynamics: Spontaneous Synchrony Breaking, Nat. Phys., 2010, vol.6, pp. 164-165.

[24] Wolfrum, M. and Omel'chenko, O. E., Chimera States Are Chaotic Transients, Phys. Rev. E, 2011, vol. 84, no. 1, 015201(R), 4 pp.

[25] Omelchenko, I., Maistrenko, Yu., Hövel, P., and Schöll, E., Loss of Coherence in Dynamical Networks: Spatial Chaos and Chimera States, Phys. Rev. Lett., 2011, vol. 106, no. 23, 234102, 4 pp.

[26] Omelchenko, I., Riemenschneider, B., Hövel, Ph., and Schöll, E., Transition from Spatial Coherence to Incoherence in Coupled Chaotic Systems, Phys. Rev. E, 2012, vol. 85, no. 2, 026212, 9 pp.

[27] Maistrenko, Yu. L., Vasylenko, A., Sudakov, O., Levchenko, R., and Maistrenko, V.L., Cascades of Multiheaded Chimera States for Coupled Phase Oscillators, Internat. J. Bifur. Chaos Appl. Sci. Engrg., 2014, vol. 24, no. 8, 1440014, 17 pp.

[28] Zakharova, A., Kapeller, M., and Schöll, E., Chimera Death: Symmetry Breaking in Dynamical Networks, Phys. Rev. Lett., 2014, vol.112, no. 15, 154101, 5 pp.

[29] Yeldesbay, A., Pikovsky, A., and Rosenblum, M., Chimeralike States in an Ensemble of Globally Coupled Oscillators, Phys. Rev. Lett., 2014, vol. 112, no. 14, 144103, 5 pp.

[30] Dudkowski, D., Maistrenko, Yu., and Kapitaniak, T., Different Types of Chimera States: An Interplay between Spatial and Dynamical Chaos, Phys. Rev. E, 2014, vol. 90, no. 3, 032920, 5 pp.

[31] Semenova, N., Zakharova, A., Schöll, E., and Anishchenko, V., Does Hyperbolicity Impede Emergence of Chimera States in Networks of Nonlocally Coupled Chaotic Oscillators?, Europhys. Lett., 2015, vol. 112, no. 4, 40002, 6 pp.

[32] Olmi, S., Martens, E. A., Thutupalli, S., and Torcini, A., Intermittent Chaotic Chimeras for Coupled Rotators, Phys. Rev. E, 2015, vol. 92, no. 3. 030901, 6 pp.

[33] Hizanidis, J., Panagakou, E., Omelchenko, I., Schöll, E., Hövel, Ph., and Provata, A., Chimera States in Population Dynamics: Networks with Fragmented and Hierarchical Connectivities, Phys. Rev. E, 2015, vol. 92, no. 1, 012915, 11 pp.

[34] Vadivasova, T.E., Strelkova, G. I., Bogomolov, S. A., and Anishchenko, V. S., Correlation Analysis of the Coherence-Incoherence Transition in a Ring of Nonlocally Coupled Logistic Maps, Chaos, 2016, vol. 26, no. 9, 093108, 9 pp.

[35] Kemeth, F. P., Haugland, S. W., Schmidt, L., Kevrekidis, I. G., and Krischer, K., A Classification Scheme for Chimera States, Chaos, 2016, vol. 26, no. 9, 094815, 8 pp.

[36] Ulonska, S., Omelchenko, I., Zakharova, A., and Schöll, E., Chimera States in Networks of van der Pol Oscillators with Hierarchical Connectivities, Chaos, 2016, vol. 26, no. 9, 094825, 9 pp.

[37] Semenova, N., Zakharova, A., Anishchenko, V., and Schöll, E., Coherence-Resonance Chimeras in a Network of Excitable Elements, Phys. Rev. Lett., 2016, vol. 117, no. 1, 014102, 6 pp.

[38] Schöll, E., Synchronization Patterns and Chimera States in Complex Networks: Interplay of Topology and Dynamics, Eur. Phys. J. Spec. Top., 2016, vol.225, nos. 6-7, pp. 891-919.

[39] Semenov, V., Zakharova, A., Maistrenko, Yu., and Schöll, E., Delayed-Feedback Chimera States: Forced Multiclusters and Stochastic Resonance, Europhys. Lett., 2016, vol. 115, no. 1, 10005, 6 pp.

[40] Sawicki, J., Omelchenko, I., Zakharova, A., and Schöll, E., Chimera States in Complex Networks: Interplay of Fractal Topology and Delay, Eur. Phys. J. Spec. Top., 2017, vol. 226, no. 9, pp. 1883-1892.

[41] Rybalova, E., Semenova, N., Strelkova, G., and Anishchenko, V., Transition from Complete Synchronization to Spatio-Temporal Chaos in Coupled Chaotic Systems with Nonhyperbolic and Hyperbolic Attractors, Eur. Phys. J. Spec. Top., 2017, vol. 226, no. 9, pp. 1857-1866.

[42] Semenova, N. I., Strelkova, G. I., Anishchenko, V.S., and Zakharova, A., Temporal Intermittency and the Lifetime of Chimera States in Ensembles of Nonlocally Coupled Chaotic Oscillators, Chaos, 2017, vol. 27, no. 6, 061102, 6 pp. 
[43] Bogomolov, S. A., Slepnev, A. V., Strelkova, G. I., Schöll, E., and Anishchenko, V.S., Mechanisms of Appearance of Amplitude and Phase Chimera States in Ensembles of Nonlocally Coupled Chaotic Systems, Commun. Nonlinear Sci. Numer. Simul., 2016, vol.43, pp. 25-36.

[44] Santos, M. S., Szezezh, J. D., Jr., Batista, A. M., Caldas, I. L., Viana, R. L., and Lopes, S. R., Recurrence Quantification Analysis of Chimera States, Phys. Lett. A, 2015, vol. 379, no. 37, pp. $2188-2192$.

[45] Zakharova, A., Semenova, N., Anishchenko, V., and Schöll, E., Time-Delayed Feedback Control of Coherence Resonance Chimeras, Chaos, 2017, vol. 27, no. 11, 114320, 9 pp.

[46] Shepelev, I. A., Bukh, A. V., Vadivasova, T. E., Anishchenko, V. S., and Zakharova, A., Double-Well Chimeras in 2D Lattice of Chaotic Bistable Elements, Commun. Nonlinear Sci. Numer. Simul., 2018, vol. 54, pp. 50-61.

[47] Hagerstrom, A. M., Murphy, T. E., Roy, R., Hövel, P., Omelchenko, I., and Schöll, E., Experimental Observation of Chimeras in Coupled-Map Lattices, Nat. Phys., 2012, vol. 8, pp. 658-661.

[48] Tinsley, M.R., Nkomo, S., and Showalter, K., Chimera and Phase Cluster States in Populations of Coupled Chemical Oscillators, Nat. Phys., 2012, vol.8, pp. 662-665.

[49] Larger, L., Penkovsky, B., and Maistrenko, Yu., Virtual Chimera States for Delayed-Feedback Systems, Phys. Rev. Lett., 2013, vol. 111, no. 5, 054103, 5 pp.

[50] Martens, E.A., Thutupalli, S., Fourrière, A., and Hallatschek, O., Chimera States in Mechanical Oscillator Networks, Proc. Natl. Acad. Sci. USA, 2013, vol.110, no. 26, pp. 10563-10567.

[51] Kapitaniak, T., Kuzma, P., Wojewoda, J., Czolczynski, K., and Maistrenko, Yu., Imperfect Chimera States for Coupled Pendula, Sci. Rep., 2014, vol.4, Art. No. 6379, 4 pp.

[52] Larger, L., Penkovsky, B., and Maistrenko, Yu., Laser Chimeras As a Paradigm for Multistable Patterns in Complex Systems, Nat. Commun., 2015, vol. 6, 7752.

[53] Watanabe, S., Strogatz, S. H., van der Zant, H. S. J., and Orlando, T. P., Whirling Modes and Parametric Instabilities in the Discrete sine-Gordon Equation: Experimental Tests in Josephson Rings, Phys. Rev. Lett., 1995, vol. 74, no. 3, pp. 379-382.

[54] Li, R. D. and Erneux, T., Bifurcation to Standing and Traveling Waves in Large Arrays of Coupled Lasers, Phys. Rev. A, 1993, vol. 49, no. 2, pp. 1301-1312.

[55] Hizanidis, J., Kouvaris, N. E., Zamora-López, G., Díaz-Guilera, A., and Antonopoulos, C. G., Chimera-Like States in Modular Neural Networks, Sci. Rep., 2016, vol. 6, Art. No. 19845, 10 pp.

[56] Rattenborg, N. C., Amlaner, C. J., and Lima, S. L., Behavioral, Neurophysiological and Evolutionary Perspectives on Unihemispheric Sleep, Neurosci. Biobehav. Rev., 2000, vol. 24, no. 8, pp. 817-842.

[57] Motter, A. E., Myers, S. A., Anghel, M., and Nishikawa, T., Spontaneous Synchrony in Power-Grid Networks, Nat. Phys., 2013, vol. 9, pp. 191-197.

[58] Nishikawa, T. and Motter, A. E., Comparative Analysis of Existing Models for Power-Grid Synchronization, New J. Phys., 2015, vol. 17, 015012, 36 pp.

[59] Boccaletti, S., Bianconi, G., Criado, R., del Genio, C.I., Gómez-Gardeñes, G., Romance, M., Sendiña-Nadal, I., Wang, Z., and Zanin, M., The Structure and Dynamics of Multilayer Networks, Phys. Rep., 2014, vol. 544, no. 1, pp. 1-122.

[60] Majhi, S., Perc, M., and Ghosh, D., Chimera States in Uncoupled Neurons Induced by a Multilayer Structure, Sci. Rep., 2016, vol. 6, Art. No. 39033, 10 pp.

[61] Maksimenko, V.A., Makarov, V.V., Bera, B.K., Ghosh, D., Dana, S.K., Goremyko, M.V., Frolov, N. S., Koronovskii, A. A., and Hramov, A. E., Excitation and Suppression of Chimera States by Multiplexing, Phys. Rev. E, 2016, vol. 94, no. 5, 052205, 9 pp.

[62] Ghosh, S., Kumar, A., Zakharova, A., and Jalan, S., Birth and Death of Chimera: Interplay of Delay and Multiplexing, Europhys. Lett., 2016, vol. 115, no. 6, 60005, 7 pp.

[63] Majhi, S., Perc, M., and Ghosh, D., Chimera States in a Multilayer Network of Coupled and Uncoupled Neurons, Chaos, 2017, vol.27, no. 7, 073109, 10 pp.

[64] Pecora, L. M. and Carroll, Th. L., Synchronization in Chaotic Systems, Phys. Rev. Lett., 1990, vol. 64 , no. 8, pp. 821-824. 
[65] Rulkov, N. F., Sushchik, M. M., Tsimring, L. S., and Abarbanel, H. D. I., Generalized Synchronization of Chaos in Directionally Coupled Chaotic Systems, Phys. Rev. E, 1995, vol. 51, no. 2, pp. 980-994.

[66] Rosenblum, M. G., Pikovsky, A.S., and Kurths, J., Phase Synchronization of Chaotic Oscillators, Phys. Rev. Lett., 1996, vol. 76, no. 11, pp. 1804-1807.

[67] Kocarev, L. and Parlitz, U., Generalized Synchronization, Predictability and Equivalence of Unidirectionally Coupled Systems, Phys. Rev. Lett., 1996, vol.76, no. 11, pp. 1816-1819.

[68] Li, C., Sun, W., and Kurths, J., Synchronization between Two Coupled Complex Networks, Phys. Rev. E, 2007, vol. 76, no. 4, 046204, 6 pp.

[69] Tang, H., Chen, L., Lu, J., and Tse, C. K., Adaptive Synchronization between Two Complex Networks with Nonidentical Topological Structures, Phys. A, 2008, vol. 387, no. 22, pp. 5623-5630.

[70] Wu, X., Zheng, W. X., and Zhou, J., Generalized outer Synchronization between Complex Dynamical Networks, Chaos, 2009, vol.19, no. 1, 013109, 9 pp.

[71] Wu, Y., Li, C., Wu, X., and Kurths, J., Generalized Synchronization between Two Different Complex Networks, Commun. Nonlinear Sci. Numer. Simul., 2012, vol.17, no.1, pp. 349-355.

[72] Andrzejak, R. G., Ruzzene, G., and Malvestio, I., Generalized Synchronization between Chimera States, Chaos, 2017, vol.27, no. 5, 053114, 6 pp.

[73] Bukh, A., Rybalova, E., Semenova, N., Strelkova, G., and Anishchenko, V., New Type of Chimera and Mutual Synchronization of Spatiotemporal Structures in Two Coupled Ensembles of Nonlocally Coupled Interacting Chaotic Maps, Chaos, 2017, vol. 27, no. 11, 111102, 7 pp. 